\section{(2) \\ BRAZIILIAN JOURNAL \\ OF MEDICAL AND BIOLOGICAL RESEARCH}

www.bjournal.com.br
ISSN 0100-879X

Volume 45 (3) 179-290 March 2012

BIOMIEDICAL SCIENCES

AND

CLINICAL INVESTIGATION

Braz J Med Biol Res, March 2012, Volume 45(3) 197-204

doi: $10.1590 / \mathrm{S} 0100-879 X 2012007500015$

Cultivation and identification of colon cancer stem cell-derived spheres from the Colo205 cell line

Ying-fei Li, Bing Xiao, San-fang Tu, Yuan-yuan Wang and Xiao-lang Zhang

The Brazilian Journal of Medical and Biological Research is partially financed by

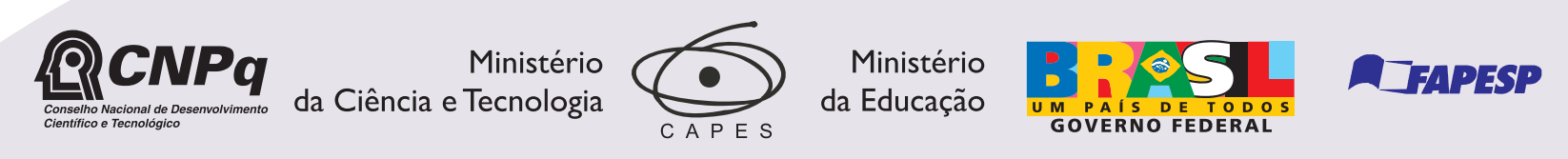

Institutional Sponsors

songo
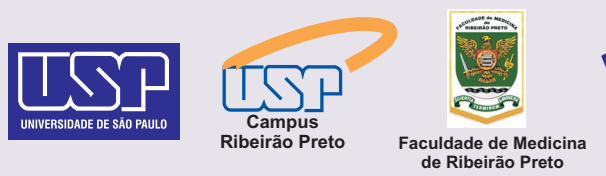

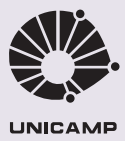

UNICAMP $\oplus$ SHIMADZU

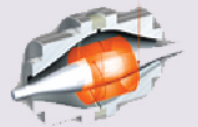

1DI Associaçāo Explore High - Performance MS Orbitrap Technology In Proteomics \& Metabolomics analitica $\underset{\text { analiticaweb.com.br }}{\text { Thermo }}$ 


\title{
Cultivation and identification of colon cancer stem cell-derived spheres from the Colo205 cell line
}

\author{
Ying-fei Li ${ }^{1,2}$, Bing Xiao' ${ }^{1}$, San-fang Tư ${ }^{3}$, Yuan-yuan Wang ${ }^{1}$ and Xiao-lang Zhang ${ }^{1}$ \\ ${ }^{1}$ Department of Gastroenterology and Institute of Gastroenterology, Nanfang Hospital, \\ Southern Medical University, Guangzhou, China \\ 2Department of Gastroenterology and Hepatology, First Municipal People's Hospital of Guangzhou, \\ Guangzhou Medical College, Guangzhou, China \\ ${ }^{3}$ Hematology Department, Zhujiang Hospital, Southern Medical University, Guangzhou, China
}

\begin{abstract}
Our group established a method to culture spheres under serum-free culture condition. However, the biological characteristics and the tumorigenicity of spheres are unknown. Here, we demonstrate that sphere cells expressed high levels of the putative colorectal cancer stem cell markers CD133 and CD44. The CD133-positive rates were 13.27 $\pm 5.62,52.71 \pm 16.97$ and 16.47 $\pm 2.45 \%$ in sphere cells, regular Colo205 cells and differentiated sphere cells, respectively, while the CD44-positive rates were $62.92 \pm 8.38,79.06 \pm 12.10$ and $47.80 \pm 2.5 \%$, respectively, and the CD133/CD44-double-positive rates were $10.77 \pm 4.96$, $46.89 \pm 19.17$ and $12.41 \pm 2.27 \%$, respectively $(P<0.05)$. Cancer sphere cells formed crypt-like structures in 3-D culture. Moreover, cells from cancer spheres exhibited more tumorigenicity than regular Colo205 cells in a xenograft assay. The cancer sphere cells displayed much higher oncogenicity than regular Colo205 cells to initiate neoplasms, as assayed by H\&E staining, Musashi-1 staining and electron microscopy. Our findings indicated that the sphere cells were enriched with cancer stem cells (CSCs), and exhibited more proliferation capacity, more differentiation potential and especially more tumorigenicity than regular Colo205 cells in vitro and in vivo. Further isolation and characterization of these CSCs may provide new insights for novel therapeutic targets and prognostic markers.
\end{abstract}

Key words: Colon cancer stem cells; Cancer sphere; Self-renew; Differentiation; Tumorigenicity

\section{Introduction}

Recent studies have shown that a small population of undifferentiated cancer cells, identifiable by the expression of special cell surface markers, possesses high differentiation potential and constant self-renewal capacity. These cancer stem cells (CSCs) are preserved and regulated by microenvironments such as tumor-associated stromal and vascular endothelial cells (1). In vivo xenotransplantations demonstrate the high tumorigenic potential of CSCs (2). In addition, CSCs express multiple drug-resistant proteins, and their greater efficiency at repairing DNA reverts damage from chemotherapy and radiation. CSCs are considered to be the root of cancer, and are responsible for cancer metastasis, recurrence and drug resistance (3). Previous studies have demonstrated CSCs in blood (4), breast (5), brain (6), pancreas (7), colon (8), liver (9), prostate (10), and skin (11) cancers. They have also been identified in several lines, such as glioma and breast cancer cell lines (12).

The Colo205 cell line was established in 1975 by Dr. Semple (13) from ascitic fluid obtained from a 70-year-old Caucasian male with colon carcinoma. In the previous study, we established a method for culturing spheres under serumfree conditions (14). However, little is known about the biology and oncogenicity of cancer spheres. In the present study, we further investigated the biological characteristics and the tumorigenicity of spheres. MTT [3-(4,5-dimethylthiazol-2-yl)-2,5-diphenyltetrazolium bromide] cell proliferation assay and 3-D culture were performed in vitro to evaluate the proliferation capacity and differentiation potential of sphere cells in comparison with regular Colo205 cells. The in vivo xenograft transplantation assay was performed to compare the tumorigenicity between sphere cells and regular Colo205 cells. Pathological analysis of xenografts

Correspondence: Bing Xiao, Department of Gastroenterology and Institute of Gastroenterology, Nanfang Hospital, Southern Medical University, 510515 Guangzhou, China. E-mail: fjxb@163.com

Received April 29, 2011. Accepted January 25, 2012. Available online February 10, 2012. Published March 19, 2012. 
was done by $\mathrm{H} \& \mathrm{E}$ staining, Musashi-1 staining and electron microscopy.

\section{Material and Methods}

\section{Culture of colon cancer spheres and differentiation assay}

Colo205 colon cancer cell lines were supplied by the American Type Culture Collection. The serum-supplemented medium (SSM) consisted of RPMI-1640 supplemented with $10 \%$ fetal calf serum. The serum-free medium (SFM) was prepared from 1:1 (v/v) Dulbecco's modified Eagle's medium and Ham's F-12 nutrient mixture (DMEM/F12; HyClone, USA), B27 supplement (1:50; Gibco, USA), 20 ng/mL epidermal growth factor (EGF; PeproTech, USA), 10 $\mathrm{ng} / \mathrm{mL}$ basic fibroblast growth factor (bFGF; PeproTech), 10 $\mathrm{ng} / \mathrm{mL}$ leukemia inhibitory factor (Chemicon, USA), and 2 $\mathrm{mM}$ L-glutamine. Colo205 cells were subcultured in SSM. Cells at the exponential growth phase were washed with PBS and digested with trypsin, followed by resuspension in SFM. Living cells were counted by Trypan blue exclusion and subcultured in SFM at a concentration of $5 \times 10^{5}$ / $\mathrm{mL}$. After cancer spheres were generated, EGF and bFGF were removed from the culture medium and $10 \%$ serum was added to induce differentiation. Cell morphology was observed with a light microscope.

\section{Cell proliferation assays}

Undifferentiated sphere cells, differentiated sphere cells and regular Colo205 cells were plated in $0.1 \mathrm{~mL}$ volumes of SFM, SSM, and SSM, respectively, at a density of 1000 cells/well in 96-well microwell plates. Cell proliferation assays were performed on days $0,2,4,6$, and 8 using the MTT method (Sigma, USA). Quantification of viable cells by measuring absorption spectra at $575 \mathrm{~nm}$ was performed with a Versamax microplate reader.

\section{Detection of the surface marker of cancer spheres}

Cells were collected separately from colon cancer spheres, post-differentiated sphere cells and regular Colo205 cells by trypsin digestion, followed by washing and resuspending in PBS at a concentration of $5 \times 10^{6} / \mathrm{mL}$. Cells were incubated with fluorescein isothyocyanate (FITC)conjugated anti-CD44 and phycoerythrin (PE)-conjugated anti-CD133 monoclonal antibodies for $30 \mathrm{~min}$ at $4^{\circ} \mathrm{C}$. The fluorescence intensity was measured by flow cytometry.

\section{3-D culture}

When typical colon cancer spheres were generated, cells were mechanically and enzymatically dissociated and resuspended in SSM, and the concentration was adjusted to $6 \times 10^{4} / \mathrm{mL}$. The regular Colo205 cells and differentiated sphere cells at the same concentrations were used as controls. 3-D culture was conducted using Matrigel matrix (BD, USA) to observe crypt-like structures. Briefly, cells were mixed 1:1 (v/v) with Matrigel and $0.5-\mathrm{mL}$ aliquots were subsequently plated onto 24 -well plates. The Matrigel was incubated at $37^{\circ} \mathrm{C}$ for $30 \mathrm{~min}$, followed by the addition of $1 \mathrm{~mL}$ SFM. The medium was changed every 3 days by replacing $0.5 \mathrm{~mL}$ spent medium with fresh medium.

\section{Subcutaneous xenograft assay in NOD/SCID mice}

All animal experiments were approved by the Animal Ethics Committee of Southern Medical University and the protocol of animal treatment was approved by the Institutional Animal Care and Use Committee. To study the tumorigenic potential of CSCs isolated from Colo205 cells, cancer spheres were mechanically and enzymatically dissociated into singlecell suspensions in PBS at concentrations of $1 \times 10^{5}, 5 \times$ $10^{5}, 1 \times 10^{6}$, and $1 \times 10^{7} / \mathrm{mL}$. Cells were mixed $1: 1(\mathrm{v} / \mathrm{v})$ with Matrigel and injected subcutaneously into one side of the flanks of NOD/SCID mice (Shanghai Si Laike Experimental Animals, China). Regular Colo205 cells were injected into the other side as a control. Fifteen days after the injection, mice were sacrificed, tumor nodules were immediately removed, fixed in $10 \%$ phosphate-buffered formalin, and embedded in paraffin. Four micrometer-thick sections were cut, mounted on poly-L-lysine-coated slides (Sigma), dried overnight at $37^{\circ} \mathrm{C}$, dewaxed in xylene, rehydrated according to standard histopathological procedure, and stained with H\&E.

To determine Musashi-1 immunoreactivity, we used heat-induced epitope retrieval methods. The antigen was retrieved with $1.0 \mathrm{mM}$ EDTA repair fluid, $\mathrm{pH}$ 8.0, via microwave heating (high power for $5 \mathrm{~min}$, followed by moderate power for $15 \mathrm{~min}$ ). Sections were incubated overnight at $4^{\circ} \mathrm{C}$ with anti-human Musashi-1 antibody (1:200; Chemicon). Immunodetection was performed using an egg proteinbiotin-horseradish peroxidase kit (Sigma).

For transmission electron microscopy, the fresh tumor was cut into $1 \times 1 \times 1-\mathrm{mm}^{3}$ cubes, fixed with fresh pre-cooled $2.5 \%$ glutaraldehyde for $72 \mathrm{~h}$ at $4^{\circ} \mathrm{C}$, followed by incubation in $1 \%$ osmium acid for $2 \mathrm{~h}$ in the dark. After dehydration with acetone, immersion and embedding, thin sections were cut and stained with uranyl acetate and lead citrate.

\section{Results}

\section{Culture of colon cancer spheres and differentiation assay}

A small proportion of Colo205 cells ( $~ 5 \%$ ) survived in SFM containing a growth factor after 7 days as measured by Trypan blue exclusion, and formed suspended colon cancer spheres. The refractile sphere cells were intensely stained, and cells were closely connected. New spheres were generated next to existing spheres, demonstrating their self-renewal capacity. Cancer spheres were passaged through at least eight generations in SFM. When the SFM was replaced with the medium containing serum, cells in the spheres migrated out and formed a single-cell layer in 10 days (Figure 1 ). 

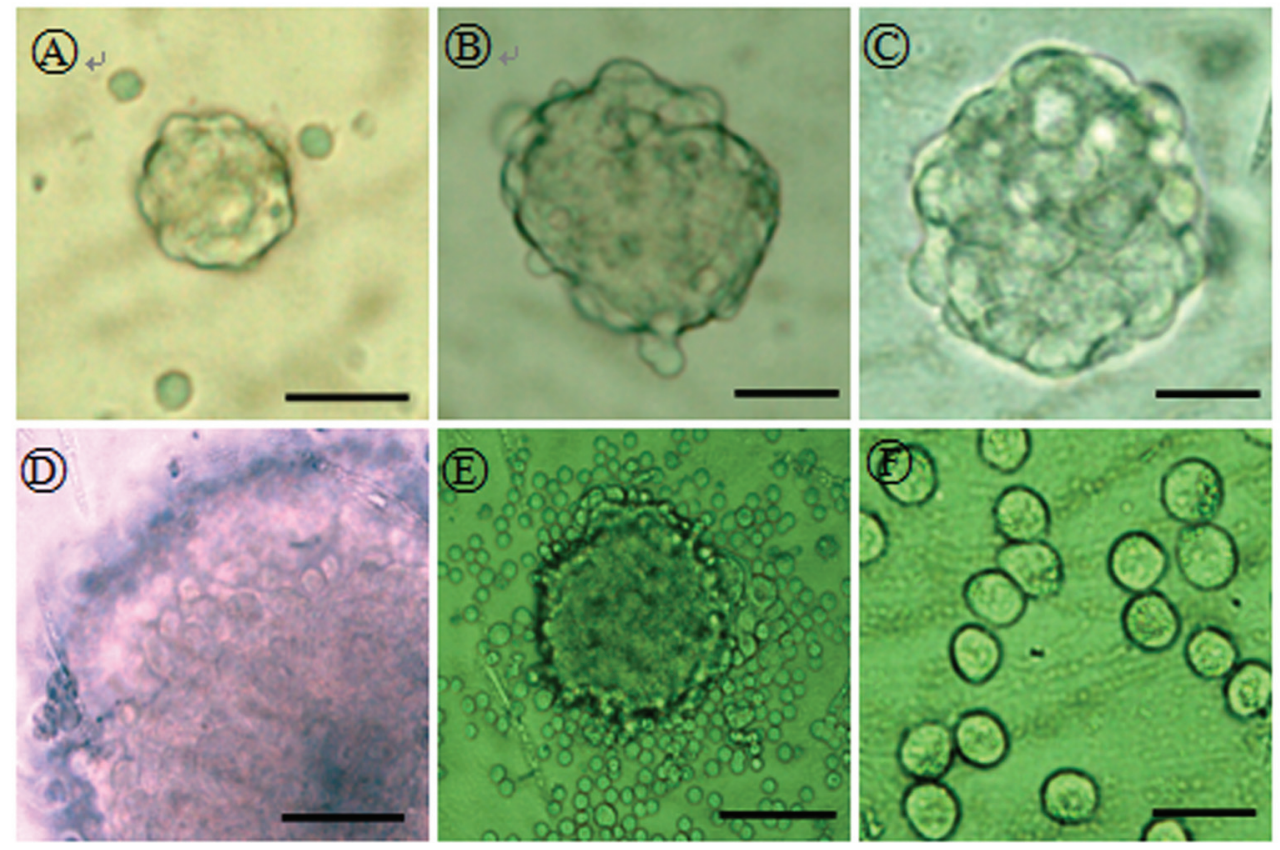

Figure 1. Cell morphology observed with an inverted microscope. $A$, Cancer sphere at first passage $B$, cancer sphere at passage 3; $C$, cancer sphere at passage 8; $D$, cells are closely connected within spheres; $E$, one day after supplementation with fetal bovine serum (FBS); $F$, ten days after supplementation with FBS. Magnification bars: $50 \mu \mathrm{m}(\mathrm{A}, \mathrm{D}) ; 25 \mu \mathrm{m}(\mathrm{B}, \mathrm{C}, \mathrm{F}) ; 100 \mu \mathrm{m}(\mathrm{E})$.

\section{Cell proliferation assays}

To evaluate the proliferative capacity of cancer sphere cells, cells were plated at 1000 cells/well, and the number of viable cells was quantified on days $0,2,4,6$, and 8 by the MTT assay. Undifferentiated sphere cells in SFM demonstrated increased proliferative capacity compared to differentiated sphere cells and regular Colo205 cells in SSM (Figure 2).

\section{Detection of the surface marker of cancer spheres}

Cells in cancer spheres expressed high levels of CD133 and CD44 in SFM (Figure 3). However, when the SFM was replaced with SSM for 10 days, the proportion of CD133 and CD44 decreased significantly (Figure 4).

\section{3-D culture}

The 3-D in vitro cell cultivation system provides the physiological signals necessary for colon cancer morphogenesis, i.e., the differentiation potential of sphere cells to recapitulate in vivo spatial orientation and crypt-like architecture. Differences between cancer spheres and the control groups were not notable on the first day. Typical crypt-like structures emerged from cancer spheres on day 18. A tubular ring interconnected structure and overlapping upward growth layers were formed in the colon cancer spheres, while the control groups did not form any crypt-like structure. These results demonstrated that colon cancer

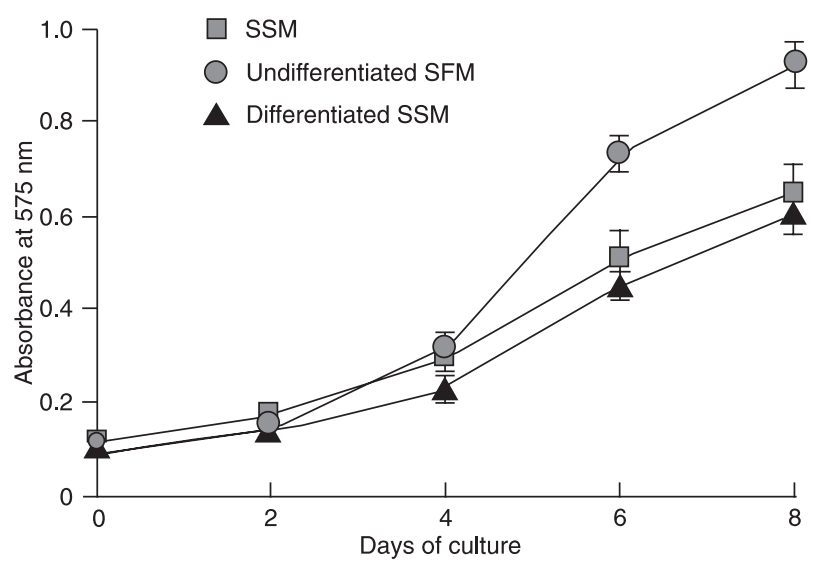

Figure 2. Cell proliferation assays showed that cancer sphere cells in serum-free medium (SFM) exhibit greater proliferation than differentiated sphere cells and regular Colo205 cells cultured in serum-supplemented medium (SSM). Data are reported as means $\pm \mathrm{SD}$.

spheres had a stronger proliferation and differentiation potential than regular Colo205 cells (Figure 5).

\section{In vivo injection of cancer sphere cells}

To assess the tumorigenicity of cancer sphere cells, 

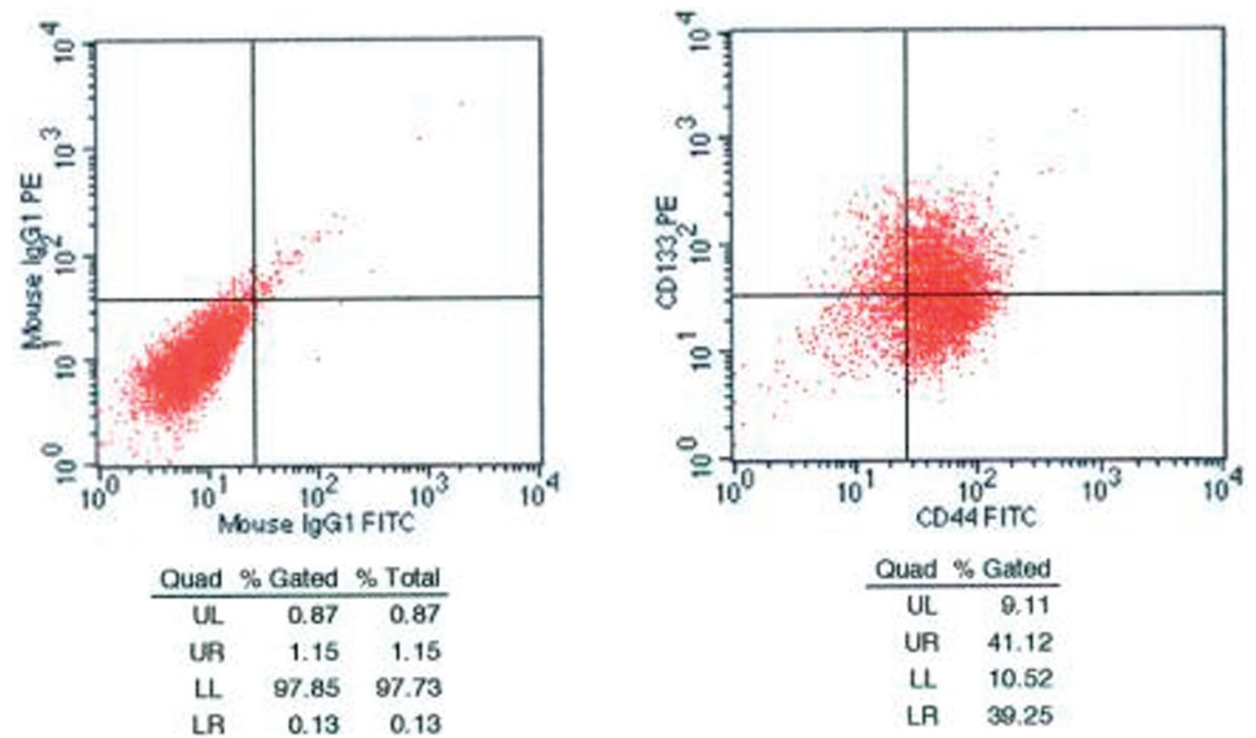

Figure 3. Flow cytometry dot plots show that cancer sphere cells expressed high levels of CD133 and CD44 in serum-free medium. Left, Isotype control. Right, Cancer sphere cells incubated with FITC-conjugated anti-CD44 and PE-conjugated anti-CD133 monoclonal antibodies. FITC = fluorescein isothyocyanate; $P E=$ phycoerythrin.

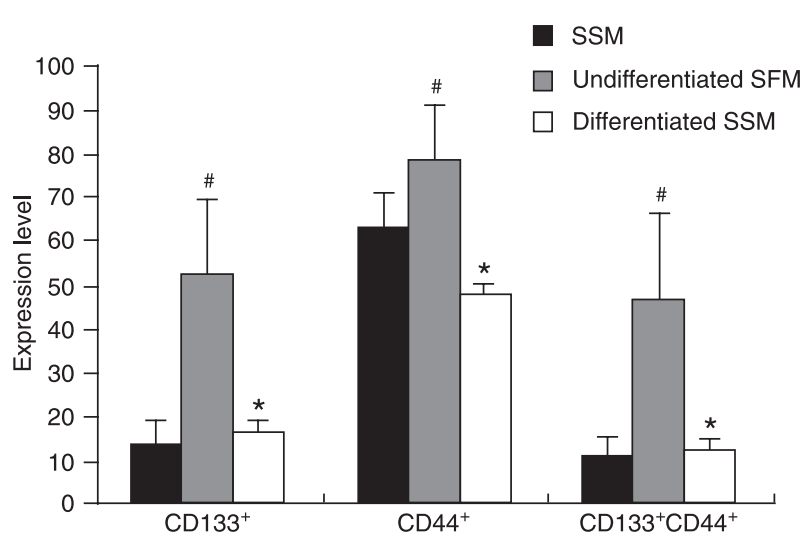

Figure 4. Expression of CD133 and CD44 in regular Colo205 cells cultured in serum-supplemented medium (SSM), undifferentiated cells from cancer spheres cultured in serum-free medium (SFM), and differentiated cells from cancer spheres cultured in SSM. Data are reported as means \pm SD. \#P $<0.05$, differentiated SSM group vs regular SSM group. ${ }^{*} \mathrm{P}>0.05$ vs regular SSM group (one-way ANOVA).

we injected the cells into NOD/SCID mice. The xenograft transplantation assay showed that $10^{4}$ colon cancer sphere cells were sufficient to induce tumorigenesis, while the same amount of regular Colo205 cells failed to induce visible tumors 15 days after injection. The volume of tumors generated by $10^{5}$ colon cancer sphere cells was significantly greater than the tumor volume of the control group, indicating that colon cancer spheres had high tumorigenicity (Table 1 and Figure 6). The histochemical assay showed that the tumors generated from sphere cells and regular Colo205 cells had a similar differentiation structure. The intestinal Musashi-1-positive stem cells were similar in the two types of tumor. Similar malignant phenotypes were also observed in transmission electron micrographs of both xenografts (Figure 7).

\section{Discussion}

A long-term study (15) reported that EGF and bFGF have a synergistic effect on proliferation of neural stem cells in vitro. Martens et al. (15) found that FGF-responsive neural stem cells cultured in SFM containing EGF and bFGF divided asymmetrically to reproduce themselves and produced EGF-responsive progenitor stem cells. The EGF-responsive population increased in size by asymmetric divisions of FGF-responsive cells and by symmetric divisions of EGF-responsive stem cells. Thus, with certain growth factors, neural stem cells could proliferate in vitro and maintain their pluripotency potential. Similar findings have been obtained in brain tumors and epithelial cancer, such as glioma (6) and colon cancer (8), respectively. Once cancer cells were cultured in SFM containing EGF and bFGF, most of the differentiated cells could not survive. However, a few undifferentiated cells, i.e., CSCs, were able to form cancer spheres and to maintain an undifferentiated state.

In the present study, only a small fraction of cells survived in SFM with EGF and bFGF, proliferated and formed suspended cancer spheres. Over a prolonged culture period, cancer spheres became larger and larger, and could 
passage into at least eight generations, evidence of their self-renewal capacity and differentiation potential.

A phenotype study was performed to analyze the
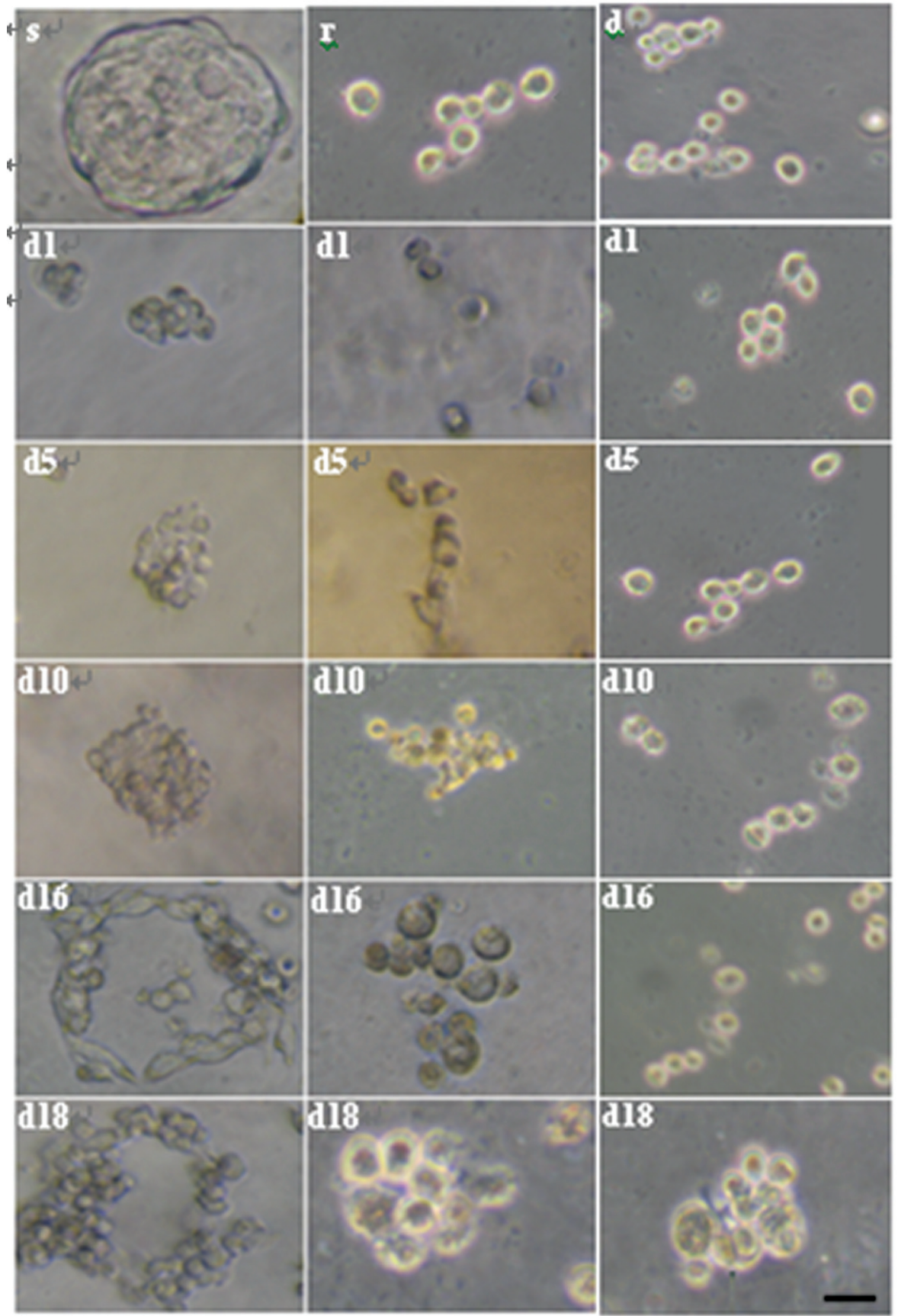

Figure 5. 3-D culture of cancer sphere cells (left panels), regular Colo205 cells (middle panels) and differentiated cells (right panels) from day 1 to day 18. Differences between cancer spheres and control groups were not notable on the first day (d1). Typical crypt-like structures emerged from cancer spheres on day 18 (left), while regular cancer cells and differentiated cells did not form any crypt-like structure (middle and right). $s=$ sphere cells; $r=$ regular cancer cells; $d=$ differentiated cells. Magnification bar: $25 \mu \mathrm{m}$. expression of CD133 and CD44 on cells from cancer spheres. CD133 is a $120-k D a$ five-transmembrane domain glycoprotein expressed on normal primitive hematopoietic, endothelial, neural, and epithelial cells. This molecule shows a highly homologous structure from nematodes to Homo sapiens. The specific function of CD133 is unclear (16). Recently, it has been considered to be a CSC surface marker for brain tumors (6), and pancreatic (7), colon (8), liver (9), and prostate (10) cancers.

CD44 is the receptor for hyaluronic acid and belongs to a type of adhesion molecule. CD44 is involved in a variety of signaling pathways, mediated cells and cell connections (17). CD44 ${ }^{+}$cancer cells are regarded as more primitive, and also as a stem cell marker for cancers of the breast (5), pancreas (7), prostate (10), head and neck squamous cell carcinoma (18), and colon $(19,20)$. CD44 ${ }^{+}$colon polyp cells have been found to express high levels of the intestinal stem cell marker Musashi-1 (21).

In the present study, the expression of CD133 and CD44 in colon cancer spheres was significantly higher than in regular Colo205 cells cultured in SSM. When SFM was replaced with SSM to induce differentiation, the expression of CD133 and CD44 in cells from cancer spheres was significantly decreased, suggesting that CD133 and CD44 were primarily expressed on undifferentiated rather than differentiated cancer cells. We concluded that there was a small population of CSCs that persisted in the Colo205 cell line. These cells formed cancer spheres in SFM and could maintain an undifferentiated stage. 
Two groups have recently reported the biology of colon cancer sphere cells in a 3-D culture system $(22,23)$. We also studied the biological characteristics of cancer spheres cultured in vitro using a Matrigel matrix. The Matrigel matrix is a solubilized basement membrane preparation extracted from mouse sarcoma, which plays a role in adhesion and differentiation of epithelial cells. Cancer spheres survived in the 3-D culture and formed crypt-like structures, while the regular Colo205 cells and post-differentiated cancer cells failed to proliferate. This suggests that cancer spheres

Table 1. In vivo tumorigenicity of cancer sphere cells and regular Colo205 cells in NOD/SCID mice.

\begin{tabular}{lcccc}
\hline & \multicolumn{4}{c}{ Cell No. } \\
\cline { 2 - 5 } & $1 \times 10^{4}$ & $5 \times 10^{4}$ & $1 \times 10^{5}$ & $1 \times 10^{6}$ \\
\hline Cancer sphere cells & $6 / 8$ & $8 / 8$ & $8 / 8$ & $8 / 8$ \\
Regular Colo205 cells & $0 / 8$ & $0 / 8$ & $6 / 8$ & $8 / 8$ \\
\hline
\end{tabular}

Cells were assayed for the ability to form tumors after subcutaneous injection into the flank of NOD/SCID mice at $1 \times 10^{4}, 5 \times 10^{4}$, $1 \times 10^{5}$, and $1 \times 10^{6}$ cells per injection. The analysis was completed 4 weeks following injection. Data are reported as number of tumors formed/number of injections. have a high differentiation potential and strong proliferation capacity. It is noteworthy that in this study the basement membrane analogues of the Matrigel matrix induced cancer differentiation, indicating the importance of the microenvironment in maintaining an undifferentiated state and the high capacity of CSCs for self-renewal. Thus, drugs targeted to a microenvironment may also be effective in killing or inducing differentiation of CSCs.

Tumorigenicity is considered to be an indispensable prerequisite for fulfilling the definition of CSCs, which should also possess self-renewal and differentiation capacities (24). The xenograft transplantation assay showed that cancer spheres are of high tumorigenicity. As few as $10^{4}$ colon cancer sphere cells induced evident cancer, while the same number of regular Colo205 cells did not show any tumorigenicity. This indicates that cancer spheres were enriched with colon CSCs, which had strong proliferative capacity. In contrast, most of the regular Colo205 cells are differentiated, with only limited proliferative capacity and therefore displayed less tumorigenicity.

$\mathrm{H} \& \mathrm{E}$ analysis showed a similar differentiation structure between the two types of xenografts. Both types expressed the intestinal stem cell marker Musashi-1 at similar levels. Similar malignant phenotypes were also found by transmission electron microscopy in both xenografts. Thus, although the degree of tumorigenicity varied greatly between the

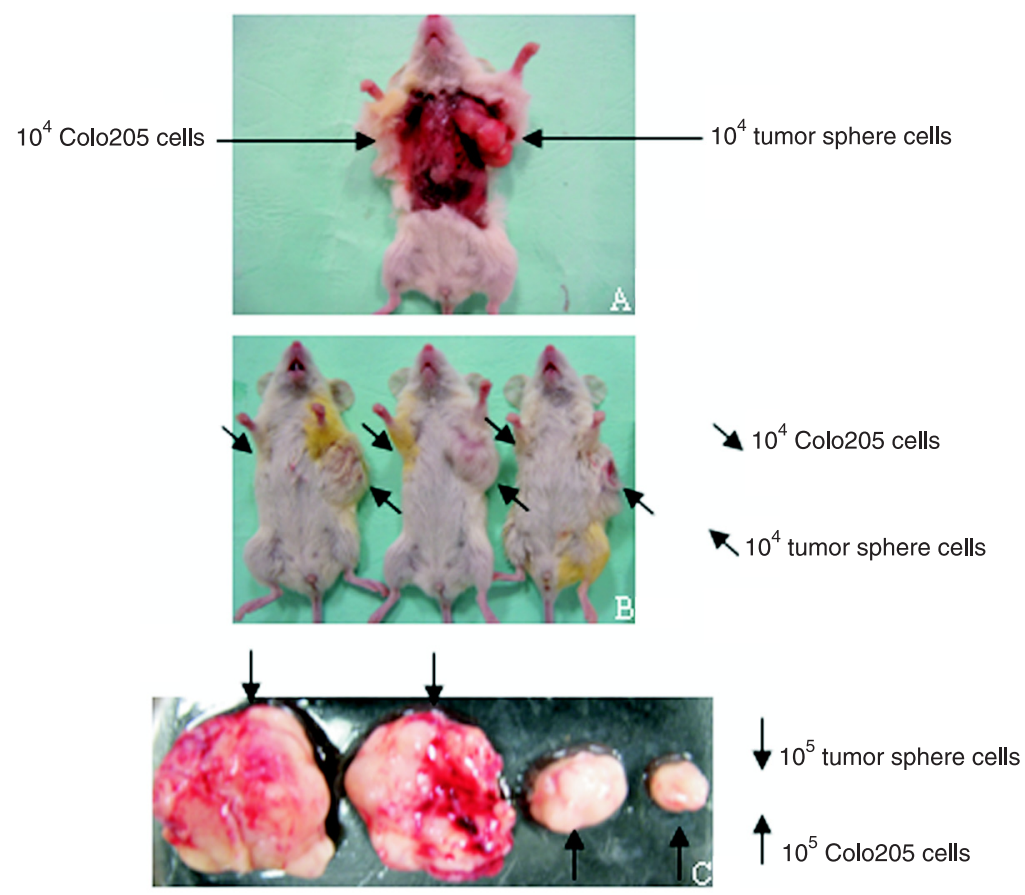

Figure 6. Immunodeficient mouse transplantation assays. $A$ and $B$, Colon cancer sphere cells $\left(10^{4}\right)$ induced neoplasms, while the same number of regular Colo205 cells did not show any tumorigenicity. $C$, Cancer sphere cells $\left(10^{4}\right)$ generated a larger neoplasm than the same number of regular cancer cells. 

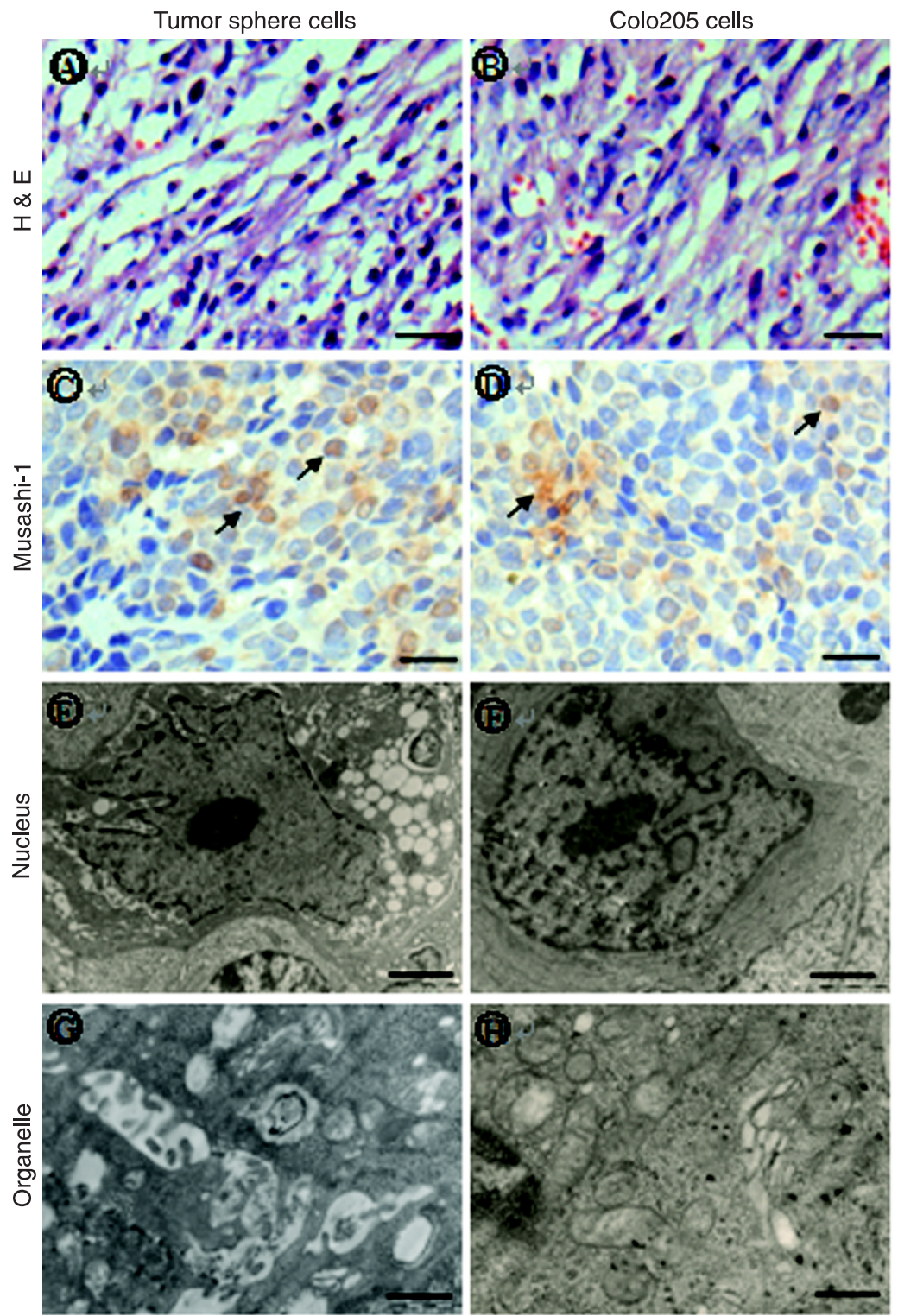

Figure 7. Histopathologic comparison of xenografts between cancer sphere groups (left panels) and regular Colo205 cell groups (right panels). $A$ and $B, H \& E$ analysis of both xenografts. $C$ and $D$, The Musashi-1-positive cells were similar between the two types of tumor, as indicated by arrows. $E$ and $F$, Similar nucleus morphologies were seen in transmission electron micrographs of both xenografts. $G$ and $H$, Organelle observed by transmission electron microscopy of both xenografts. Magnification bars: $25 \mu \mathrm{m}(\mathrm{A}, \mathrm{B}, \mathrm{C}, \mathrm{D}) ; 2 \mu \mathrm{m}(\mathrm{E}, \mathrm{F}) ; 1 \mu \mathrm{m}(\mathrm{G}, \mathrm{H})$.

cancer spheres and regular Colo205 cells, the phenotypes of tumors generated from these two groups of cells were the same.

In summary, the sphere cells exhibited more proliferation capacity and more differentiation potential in in vitro cell proliferation assays and 3-D culture assays. Moreover, cancer sphere cells displayed more tumorigenicity than regular Colo205 cells in xenograft transplantation assays.
Our findings may provide new insights into the tumorigenesis of colon cancer and offer a potential target for anti-cancer therapy.

\section{Acknowledgments}

The authors thank Medjaden Bioscience Limited for assisting with the preparation of this manuscript. 


\section{References}

1. Gilbert CA, Ross AH. Cancer stem cells: cell culture, markers, and targets for new therapies. J Cell Biochem 2009; 108: 1031-1038.

2. Ferrand A, Sandrin MS, Shulkes A, Baldwin GS. Expression of gastrin precursors by CD133-positive colorectal cancer cells is crucial for tumour growth. Biochim Biophys Acta 2009; 1793: 477-488.

3. Ward RJ, Dirks PB. Cancer stem cells: at the headwaters of tumor development. Annu Rev Pathol 2007; 2: 175-189.

4. Lapidot T, Sirard C, Vormoor J, Murdoch B, Hoang T, Caceres-Cortes $\mathrm{J}$, et al. A cell initiating human acute myeloid leukaemia after transplantation into SCID mice. Nature 1994; 367: 645-648.

5. Al-Hajj M, Wicha MS, Benito-Hernandez A, Morrison SJ, Clarke MF. Prospective identification of tumorigenic breast cancer cells. Proc Natl Acad Sci U S A 2003; 100: 39833988.

6. Singh SK, Clarke ID, Terasaki M, Bonn VE, Hawkins C, Squire $\mathrm{J}$, et al. Identification of a cancer stem cell in human brain tumors. Cancer Res 2003; 63: 5821-5828.

7. Lee CJ, Dosch J, Simeone DM. Pancreatic cancer stem cells. J Clin Oncol 2008; 26: 2806-2812.

8. Ricci-Vitiani L, Lombardi DG, Pilozzi E, Biffoni M, Todaro $\mathrm{M}$, Peschle $\mathrm{C}$, et al. Identification and expansion of human colon-cancer-initiating cells. Nature 2007; 445: 111-115.

9. Suetsugu A, Nagaki M, Aoki H, Motohashi T, Kunisada T, Moriwaki H. Characterization of CD133+ hepatocellular carcinoma cells as cancer stem/progenitor cells. Biochem Biophys Res Commun 2006; 351: 820-824.

10. Collins AT, Berry PA, Hyde C, Stower MJ, Maitland NJ. Prospective identification of tumorigenic prostate cancer stem cells. Cancer Res 2005; 65: 10946-10951.

11. Schatton T, Murphy GF, Frank NY, Yamaura K, WaagaGasser AM, Gasser M, et al. Identification of cells initiating human melanomas. Nature 2008; 451: 345-349.

12. Setoguchi T, Taga T, Kondo T. Cancer stem cells persist in many cancer cell lines. Cell Cycle 2004; 3: 414-415.

13. Semple TU, Quinn LA, Woods LK, Moore GE. Tumor and lymphoid cell lines from a patient with carcinoma of the colon for a cytotoxicity model. Cancer Res 1978; 38: 1345-1355.

14. Li YF, Xiao B, Lai ZS, Tu SF, Wang YY, Zhang XL. [Spheres isolated from Colo205 cell line possess cancer stem-like cells under serum-free culture condition]. Nan Fang Yi Ke Da Xue Xue Bao 2008; 28: 236-240.

15. Martens DJ, Tropepe V, van Der Kooy D. Separate proliferation kinetics of fibroblast growth factor-responsive and epidermal growth factor-responsive neural stem cells within the embryonic forebrain germinal zone. J Neurosci 2000; 20: 1085-1095.

16. Mizrak D, Brittan M, Alison MR. CD133: molecule of the moment. J Pathol 2008; 214: 3-9.

17. Schmidt DS, Klingbeil P, Schnolzer M, Zoller M. CD44 variant isoforms associate with tetraspanins and EpCAM. Exp Cell Res 2004; 297: 329-347.

18. Prince ME, Sivanandan R, Kaczorowski A, Wolf GT, Kaplan MJ, Dalerba P, et al. Identification of a subpopulation of cells with cancer stem cell properties in head and neck squamous cell carcinoma. Proc Natl Acad Sci U S A 2007; 104: 973978.

19. Dalerba P, Dylla SJ, Park IK, Liu R, Wang X, Cho RW, et al. Phenotypic characterization of human colorectal cancer stem cells. Proc Natl Acad Sci U S A 2007; 104: 1015810163.

20. Horst D, Kriegl L, Engel J, Kirchner T, Jung A. Prognostic significance of the cancer stem cell markers CD133, CD44, and CD166 in colorectal cancer. Cancer Invest 2009; 27: 844-850.

21. Schulenburg A, Cech P, Herbacek I, Marian B, Wrba F, Valent $P$, et al. CD44-positive colorectal adenoma cells express the potential stem cell markers musashi antigen (msi1) and ephrin B2 receptor (EphB2). J Pathol 2007; 213: 152-160.

22. Vermeulen L, Todaro M, de Sousa Mello F, Sprick MR, Kemper K, Perez Alea M, et al. Single-cell cloning of colon cancer stem cells reveals a multi-lineage differentiation capacity. Proc Natl Acad Sci U S A 2008; 105: 13427-13432.

23. Yeung TM, Gandhi SC, Wilding JL, Muschel R, Bodmer WF. Cancer stem cells from colorectal cancer-derived cell lines. Proc Natl Acad Sci U S A 2010; 107: 3722-3727.

24. Clarke MF, Dick JE, Dirks PB, Eaves CJ, Jamieson CH, Jones DL, et al. Cancer stem cells - perspectives on current status and future directions: AACR Workshop on cancer stem cells. Cancer Res 2006; 66: 9339-9344. 